\title{
Utilisation de la barymétrie pour l'estimation du poids chez le zébu Azawak au Niger
}

\author{
K. Dodo ${ }^{1,2}$ V.S. Pandey ${ }^{2 *}$ M.S. Illiassou ${ }^{3}$
}

\begin{abstract}
Mots-clés
Bovin - Zébu Azawak - Mensuration corporelle - Poids corporel - Niger.
\end{abstract}

\begin{abstract}
Résumé
L'objectif de cette étude a été de déterminer des équations barymétriques applicables au zébu Azawak. Pour cela, des pesées et 12 types de mensurations ont été effectuées chez 343 zébus Azawak pendant trois mois, à la station sahélienne expérimentale de Toukounous au Niger. Les animaux étaient essentiellement jeunes (74 p. 100 avaient au plus deux ans d'âge). Parmi les 12 types de mensurations, trois (le périmètre thoracique, la hauteur au garrot et la longueur scapulo-ischiale) ont été retenues pour établir la barymétrie en raison de leur corrélation avec le poids. La corrélation du poids avec le périmètre thoracique $(r=0,97)$ ou avec la longueur scapulo-ischiale $(r=0,96)$ a été plus élevée qu'avec la hauteur au garrot $(r=0,92)$. Plusieurs types de liaisons entre le poids et les deux types de mensurations (périmètre thoracique et longueur scapulo-ischiale) ont été comparées. L'équation barymétrique retenue pour l'ensemble des animaux (mâles et femelles) a été une régression polynomiale du poids prédit $(\mathrm{y})$ sur le périmètre thoracique $(\mathrm{x})$ et la longueur scapulo-ischiale $(z): y=0,02697 x^{2}-4,25 x+1,535 z+96,145$ avec un coefficient de détermination $r^{2}=0,989$ et un écart-type résiduel $\left(S_{y}\right)$ de $11,23 \mathrm{~kg}$. L'erreur entre le poids observé et le poids prédit, exprimée en pourcentage du poids observé, a été inférieure à 5 p. 100 chez 57,2 p. 100 des animaux et inférieure à 10 p. 100 chez 90,1 p. 100 d'entre eux. Pour les mâles, une équation également à deux variables a été proposée : $y=0,0260 x^{2}-3,856 x+$ $1,211 z+96,114$ avec $r^{2}=0,990$ et $S_{y}=10,33 \mathrm{~kg}$. Chez les femelles, une régression à partir du périmètre thoracique uniquement $\left(\mathrm{y}=0,0281 \mathrm{x}^{2}-\right.$ $3,294 x+125,217)$ a aussi permis d'obtenir une bonne précision $\left(r^{2}=0,981\right.$ et $S_{y}=15,28 \mathrm{~kg}$ ). Cette dernière équation a été utilisée pour établir une table de conversion du périmètre thoracique en poids.
\end{abstract}

\section{INTRODUCTION}

Le Niger est un pays intertropical à vocation essentiellement agropastorale. Après l'agriculture, l'élevage constitue la seconde activité socio-économique de la population. Le bétail se compose principalement de bovins et de petits ruminants. Le cheptel bovin nigérien est dominé par les zébus qui comprennent le zébu Azawak $(9,13)$.

Le zébu Azawak est surtout réputé pour sa bonne aptitude laitière. Sa production totale varie de 800 à $1100 \mathrm{~kg}$ de lait pour une durée

\footnotetext{
1. Ministère des Ressources animales, BP 12 091, Niamey, Niger

2. Institut de médecine tropicale Prince Léopold, Nationalestraat 155, B 2000 Antwerpen, Belgique

3. Direction des Centres de multiplication et stations d'élevage, BP 827 Niamey, Niger

* Auteur pour la correspondance

Fax : +32(0)2 2518436 ou +32(0)32161431 ; e-mail : vpandey@itg.be
}

de lactation de 270 à 300 jours, avec un lait à 4,3 p. 100 de matière grasse. Il présente aussi une bonne docilité à la traite $(1,2)$. Le zébu Azawak a bénéficié depuis longtemps d'une attention zootechnique particulière. Il a subi une sélection à la station sahélienne expérimentale de Toukounous au Niger depuis 1954. Cette amélioration génétique a visé la production d'animaux à robe fauve uniforme, aux extrémités et à la muqueuse noire, présentant de bonnes aptitudes bouchères et surtout laitières (1). La sélection du zébu Azawak au sein de cette structure étatique à Toukounous a eu pour objectif de purifier la race, de la multiplier et de la diffuser en milieu villageois.

Les interventions centrées sur l'élevage traditionnel doivent conduire à des contrôles de l'évolution pondérale des bovins, particulièrement celle des jeunes en croissance. L'emploi de bascules pèse-bétail nécessite un véhicule, du personnel et certains aménagements. Il s'avère peu pratique et coûteux (10). Une solution alternative consiste à utiliser la barymétrie. 
La barymétrie est la détermination approximative du poids vif des animaux par des mensurations (11). Cette méthode d'estimation n'a pas la rigueur d'une pesée. Elle est cependant utile car elle ne nécessite qu'un matériel léger, un minimum de personnel et une contention réduite $(7,10,11)$. De plus, une précision satisfaisante peut être attendue de l'emploi de la barymétrie chez les animaux en croissance (10).

Les formules barymétriques doivent être établies en fonction de la race, du sexe et de l'âge (16). Elles ont été déterminées chez plusieurs races de zébus et de taurins rencontrés en Afrique de l'Ouest, par exemple chez les zébus Maure et Peul (5), Kapsiki (6) et N'Dama $(8,15)$. Cela ne semble pas être le cas pour le zébu Azawak. L'objectif de cette étude a ainsi été de déterminer des équations barymétriques applicables au zébu Azawak.

\section{MATERIEL ET METHODES}

\section{Milieu de l'étude}

Le travail a été réalisé à la station sahélienne expérimentale de Toukounous qui se trouve dans la vallée de l'Azawak. Cette zone est constituée d'un plateau ondulé dont l'altitude moyenne varie de 500 à $550 \mathrm{~m}$. La station est située à $18 \mathrm{~km}$ au nord-est de Filingué et à 200 km de Niamey (14 31' de latitude N, $3^{\circ} 18^{\prime}$ de longitude O) dans la zone climatique sahélienne aride. Elle couvre une superficie de 4474 ha dont 75 p. 100 sont des sols dunaires à relief ondulé peu prononcé ; le reste est occupé par des dépressions argileuses plus ou moins inondées et impraticables en période pluvieuse. Le climat est de type sahélien, caractérisé par une longue saison sèche (d'octobre à mai) et une courte saison pluvieuse (de juin à septembre). La pluviométrie est très variable et faible, la moyenne annuelle de 1968 à 1993 ayant été de 301 mm (1).

\section{Animaux}

\section{Organisation des troupeaux}

La partie expérimentale de l'étude a été réalisée de décembre 1997 à mars 1998 (saison sèche fraîche et début de la saison sèche chaude). Au début de celle-ci, la station comptait un effectif de 606 bovins, tous de race Azawak sélectionnée. Ces animaux étaient répartis en sept troupeaux distincts : les mâles (veaux sevrés, taurillons et taureaux), les vaches suitées multipares (option lait et option viande), les vaches suitées primipares, les génisses et les vaches testées sur la descendance, les vaches et les génisses gestantes, les velles sevrées, les veaux et les velles à la mamelle (des multipares et des primipares)

Dans le cadre de ce travail, 343 bovins (soit 56,6 p. 100 de l'effectif total de la station), 114 mâles et 229 femelles de moins de
24 mois d'âge, ont été manipulés (tableau I). Les vaches suitées, et les vaches et les génisses en gestation ont été épargnées.

\section{Alimentation}

L'alimentation des animaux a été basée sur l'exploitation des pâturages naturels des 30 parcelles de la station. Au niveau des pâturages, la strate herbacée a été dominée par Schœnefeldia gracilis, Aristida mutabilis et Cenchrus biflorus, tandis que les ligneux dominants ont été Maerua crassifolia, Salvadora persica, Balanites aegyptiaca, Boscia senegalensis et Acacia sp. L'apport des ligneux fourragers a été très important dans l'alimentation des animaux, particulièrement en saison sèche. La supplémentation alimentaire a consisté en une distribution en masse de graines de coton. Elle n'a concerné que les vaches en lactation et les veaux à la mamelle à raison de $2 \mathrm{~kg}$ par vache par jour et $1 \mathrm{~kg}$ par veau par jour. Une complémentation minérale sous forme de blocs à lécher a été assurée une fois par mois. L'abreuvement s'est fait une fois par jour.

\section{Soins vétérinaires}

Les animaux de plus de six mois d'âge ont été vaccinés contre la peste bovine et la péripneumonie contagieuse des bovidés. Tous les animaux ont été vaccinés contre la pasteurellose et les charbons symptomatique et bactéridien. Pendant la saison des pluies, les animaux avaient subi un déparasitage interne avec du thiabendazole $\left(\right.$ Thibenzole ${ }^{\circledR}$ ). Le déparasitage externe a été hebdomadaire pendant la saison de pluies et a été effectué tous les 15 jours pendant la saison sèche, par aspersion avec de l'amitraz liquide (Taktic ${ }^{\circledR}$ ).

\section{Matériel et équipement}

Un parc couloir en béton a servi pour la contention des animaux sevrés ou adultes. Une bascule pèse-bétail d'une capacité de 50 à $1500 \mathrm{~kg}$ et d'une sensibilité de $1 \mathrm{~kg}$ a été utilisée pour la pesée des animaux sevrés ou adultes. Une bascule mobile d'une portée maximale de $300 \mathrm{~kg}$ a été affectée aux jeunes à la mamelle. Deux toises graduées en centimètres et des rubans métriques (de 1,50 $\mathrm{m}$ et $3 \mathrm{~m}$ ) ont servi pour les mensurations. Pour la mesure du périmètre thoracique, une sorte d'œse a été confectionnée et a permis de récupérer avec plus d'aisance le mètre ruban sous l'animal. Le matériel de contention des animaux adultes a été constitué de cordes.

\section{Méthodes}

\section{Mensurations et pesées}

Douze mensurations ont été réalisées conformément aux techniques normalisées $(9,12,13,14)$. Toutes les mensurations ont été faites avec des rubans métriques sauf la hauteur au garrot qui a été réalisée à l'aide de toises. Parmi ces douze mensurations, trois ont été retenues pour établir la barymétrie :

\section{Tableau I}

Fréquence et nombre d'observations réalisées chez les mâles et les femelles zébus Azawak selon différentes classes d'âge

$\begin{array}{lcccccc}\text { Classes d'âge } & \text { Mâles } & \text { Femelles } & \begin{array}{c}\text { Mâles et } \\ \text { Femelles }\end{array} & \text { Fréquence } & \begin{array}{c}\text { Observations } \\ \text { par animal }\end{array} & \text { Nb. d'observations } \\ \text { 0-3 mois } & 19 & 20 & 39 & 1 \text { fois/semaine } & 10 & 390 \\ \text { 3-6 mois } & 21 & 32 & 53 & 1 \text { fois/quinzaine } & 6 & 318 \\ \text { 6-24 mois } & 53 & 109 & 162 & 1 \text { fois/mois } & 2 & 324 \\ \geq 24 \text { mois } & 21 & 68 & 89 & 1 \text { fois/trimestre } & 1 & 89 \\ \text { Total } & 114 & 229 & 343 & - & - & 1121\end{array}$


- le périmètre thoracique (tour de poitrine immédiatement en arrière des épaules) ;

- la hauteur au garrot (distance verticale entre le sol et le sommet du garrot, immédiatement en arrière de la bosse, sur le sommet du scapulum et non pas sur les apophyses épineuses des vertèbres dorsales) ;

- la longueur scapulo-ischiale (de la pointe de l'épaule à la pointe de la fesse).

Les pesées ont eu lieu dans la matinée, avant l'abreuvement des animaux et immédiatement après les mensurations. Elles ont été relevées au moment où l'animal s'était immobilisé à l'intérieur de la bascule.

\section{Fréquence de la collecte des données}

Les mensurations et les pesées ont été réalisées de façon périodique selon l'âge des animaux qui a été déterminé à partir des numéros d'identification (boucles) et l'exploitation des registres de naissance ou des fiches individuelles. La fréquence adoptée (tableau I) a été préconisée ou appliquée par plusieurs auteurs (6, $9,15,16)$.

Au total, 1121 observations ont été faites sur 343 animaux (114 mâles et 229 femelles) qui ont pu être régulièrement suivis (tableau I). Il s'agissait essentiellement d'animaux jeunes. Sur les 343 animaux retenus, 254 (soit 74 p. 100) avaient moins de deux ans d'âge. Parmi les animaux, 18 ont eu un poids situé au-delà de la moyenne, plus ou moins trois fois l'écart-type. Ces données ont été considérées comme des valeurs aberrantes (en anglais, outliers) et ont été éliminées des analyses statistiques qui ont finalement porté sur 325 animaux (110 mâles et 215 femelles).

\section{Analyses statistiques}

Les moyennes et les déviations standard du poids et de toutes les mesures ont été calculées avec le logiciel Excel 7.0. Les formules barymétriques ont été déterminées grâce aux logiciels Spss 7.5 et Ncss en utilisant la méthode de régression multiple. Le poids estimé a été considéré comme la variable dépendante. Les variables indépendantes ont été le périmètre thoracique, la hauteur au garrot et la longueur scapulo-ischiale. Les différentes équations ont été comparées sur la base de leur coefficient de détermination $\left(\mathrm{r}^{2}\right)$ et de leur écart-type résiduel $\left(\mathrm{S}_{\mathrm{y}}\right)$.

\section{RESULTATS}

\section{Corrélations entre poids et mesures linéaires}

Chez tous les animaux (mâles et femelles), le poids a évolué dans le même sens et à peu près à la même allure que le périmètre thoracique, la hauteur au garrot et la longueur scapulo-ischiale (figures 1 et 2). Les corrélations entre le poids et les mesures linéaires ont toutes été significatives $(p<0,001)$. Une matrice de corrélation simple concernant les quatre variables a été établie. Les corrélations entre les quatre variables se sont situées entre 0,923 et 0,972 . Les différentes mensurations ont été fortement corrélées au poids. La corrélation a été plus marquée avec le périmètre thoracique $(r=0,972)$ et la longueur scapulo-ischiale $(r=0,963)$ qu'avec la hauteur au garrot $(0,923)$.

\section{Choix d'une formule}

Le périmètre thoracique et la longueur scapulo-ischiale ont été retenus pour la détermination de formules barymétriques en raison de leur corrélation plus élevée avec le poids. Plusieurs types de liaisons entre le poids $(\mathrm{y})$, le périmètre thoracique $(\mathrm{x})$ et la longueur scapulo-ischiale (z) ont été comparés. Les équations qui ont présenté une meilleure précision ont été des régressions polynomiales établies en fonction des variables $\mathrm{x}$ et $\mathrm{z}$. Ces formules ont eu un coefficient de détermination plus élevé et un écart-type résiduel plus bas que celles déterminées uniquement avec x (tableau II). Chez les mâles et les femelles, le poids prédit avec la régression polynomiale de y sur $\mathrm{x}$ et $\mathrm{z}$ a été très proche du poids observé. L'erreur entre le poids observé et le poids prédit, exprimée en pourcentage du poids observé (erreur relative) a été inférieure à 5 p. 100 chez 57,2 p. 100 des animaux et inférieure à 10 p. 100 chez 90,2 p. 100 d'entre eux (tableau III).

Les erreurs absolues (différences entre poids observés et poids prédits) les plus fréquentes n'ont généralement pas dépassé $3 \mathrm{~kg}$ de poids vif et il n'y a pas eu de tendance marquée pour une sous-estimation ou une surestimation du poids prédit (figure 3).

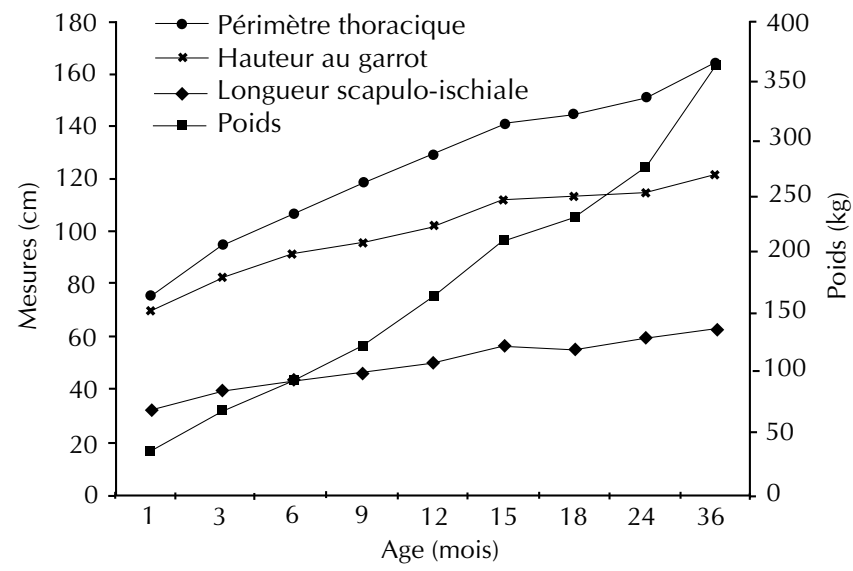

Figure 1 : évolution des mesures linéaires et du poids chez les femelles $(n=215)$.

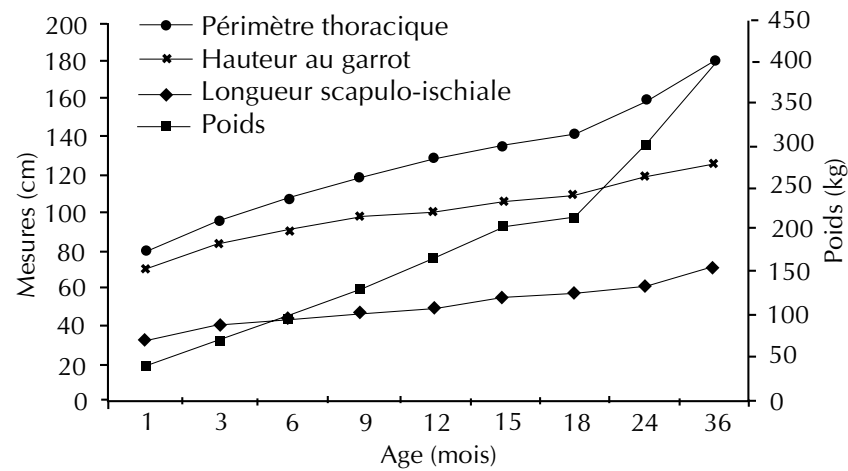

Figure 2 : évolution des mesures linéaires et du poids chez les mâles $(n=110)$.

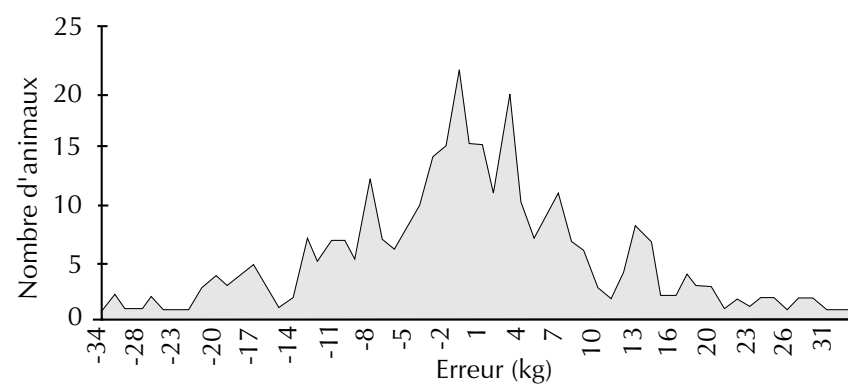

Figure 3 : fréquence des erreurs absolues entre poids prédits et poids observés $(n=325)$. 
Chez les mâles, la régression avec les deux variables $\mathrm{x}$ et $\mathrm{z}$ a été encore plus précise $\left(\mathrm{r}^{2}=0,990\right.$ et $\mathrm{S}_{\mathrm{y}}=10,33 \mathrm{~kg}$ ) (tableau II). Chez les femelles, la régression polynomiale à partir uniquement du périmètre thoracique, caractérisée par un coefficient de détermination élevé $\left(\mathrm{r}^{2}=0,981\right)$, a été retenue pour la réalisation de la courbe d'évolution du poids en fonction du périmètre thoracique (figure 4).

\section{DISCUSSION}

Le périmètre thoracique, la longueur scapulo-ischiale, la hauteur au garrot et le tour spiral sont les mensurations utilisées en barymétrie en raison de leur corrélation avec le poids. Bien que le tour spiral soit intéressant, son exécution nécessite une contention parfaite et une manipulation par deux opérateurs possédant de bonnes connaissances de la morphologie des animaux $(10,16)$.

Au niveau de l'ensemble des animaux, le poids vif a été fortement corrélé aux trois types de mensurations. Toutefois, cette corrélation a été moins marquée avec la hauteur au garrot, conformément aux conclusions de certains auteurs $(5,6,8)$. La mensuration ayant apporté le plus de précision sur la valeur prédictive du poids vif a été la mesure du périmètre thoracique, comme cela a été rapporté à l'unanimité par différents auteurs $(6,8,10,15,16)$.

La régression polynomiale du poids sur le périmètre thoracique et la longueur scapulo-ischiale s'est révélée être l'équation prédictive la plus précise pour l'ensemble des animaux. Du point de vue strictement mathématique, le coefficient de la corrélation totale de la régression multiple de y sur $\mathrm{x}$ et $\mathrm{z}$ est toujours supérieur ou égal à celui de la régression simple sur une des deux variables. Cela indique que l'information apportée par deux variables est plus riche que celle fournie par une seule (10).
La formule barymétrique sous forme de régression polynomiale proposée pour l'ensemble des animaux chez le zébu Azawak dans cette étude a révélé des erreurs peu importantes (tableau III). Des formules barymétriques sous forme d'équations linéaires avaient été proposées pour le zébu Maure (5) et le taurin N'Dama (8). Chez les animaux de 12 mois, l'erreur sur le poids estimé a été située dans la tranche de 2 p. 100 du poids observé chez 13 à 20 p. 100 des animaux, 5 p. 100 chez 37 à 43 p. 100 d'entre eux et 10 p. 100 chez 60 à 77 p. 100 d'entre eux. A 24 mois, les valeurs correspondantes ont été trouvées chez 17 à 23 p. 100, 41 à 51 p. 100 et 70 à 81 p. 100 des animaux. Dans l'ensemble, l'erreur dans l'estimation du poids par les équations linéaires s'avère plus importante que par les régressions polynomiales proposées dans cette étude. Selon Symoens et Hounsou chez le bétail Borgou, les poids en dessous de $150 \mathrm{~kg}$ et au-dessus de $250 \mathrm{~kg}$, prédits par les équations polynomiales, sont plus proches des poids observés que ceux calculés par une équation linéaire (18).

Chez les mâles, la régression polynomiale avec les deux variables s'est montrée particulièrement plus précise pour la prédiction du poids vif des animaux $\left(\mathrm{r}^{2}=0,990\right)$. Le périmètre thoracique et la longueur scapulo-ischiale (ensemble) peuvent traduire un aspect de volume et une représentation grossière des animaux sous forme de cylindre. Cette forme cylindrique serait plus prononcée chez les mâles que chez les femelles. Une liaison étroite pourrait être imaginée entre ce volume et le poids. Dans la pratique, la mesure de la longueur scapulo-ischiale est relativement facile. La très grande précision apportée par cette mensuration pourrait justifier son recours chez le zébu Azawak mâle en plus du périmètre thoracique.

Pour les femelles, une régression polynomiale du poids sur le périmètre thoracique a aussi présenté une grande précision. Ce type

\section{Tableau II}

Comparaison entre les différents types d'équations

\begin{tabular}{|c|c|c|c|}
\hline Sexe & Equations & $r^{2}$ & $\mathrm{~S}_{\mathrm{y}}$ \\
\hline Mâles et Femelles $(n=325)$ & $\begin{array}{l}y=0,02697 x^{2}-4,250 x+1,535 z+96,145 \\
y=0,00200782 x^{2}+1,3114 x-24,1225 \\
y=1,55302 x+0,0895857 z-0,00069865 x z-31,6459\end{array}$ & $\begin{array}{l}0,989 \\
0,920 \\
0,940\end{array}$ & $\begin{array}{l}11,228 \\
30,282 \\
26,225\end{array}$ \\
\hline Mâles $(n=110)$ & $y=0,0260 x^{2}-3,856 x+1,211 z+96,114$ & 0,990 & 10,328 \\
\hline Femelles $(n=215)$ & $y=0,0281 x^{2}-3,294 x+125,217$ & 0,981 & 15,279 \\
\hline
\end{tabular}

$\mathrm{y}=$ poids estimé $(\mathrm{kg})$; $\mathrm{x}$ = périmètre thoracique $(\mathrm{cm}) ; \mathrm{z}=$ longueur scapulo-ischiale $(\mathrm{cm})$;

$\mathrm{S}_{\mathrm{y}}=$ écart-type résiduel $(\mathrm{kg}) ; \mathrm{r}^{2}=$ coefficient de détermination

\section{Tableau III}

Fréquence des erreurs relatives entre poids prédits et poids observés au niveau des 325 animaux étudiés

\begin{tabular}{llrcc} 
Erreur & $\mathbf{0 - 2}$ & $\mathbf{2 - 5}$ & $\mathbf{5 - 1 0}$ & $\boldsymbol{>} \mathbf{1 0}$ \\
\hline Nb. d'animaux & \multicolumn{1}{c}{77} & \multicolumn{1}{c}{109} & \multicolumn{1}{c}{107} & \multicolumn{1}{c}{32} \\
Fréquence $(\%)$ & 23,69 & 33,54 & 32,92 & 9,85 \\
Ecart moyen $(\mathrm{kg})$ & 6,01 & 6,90 & 10,89 & 13,62
\end{tabular}

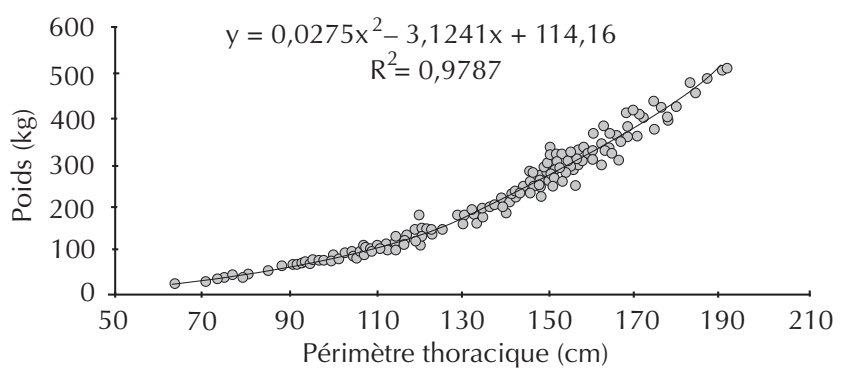

Figure 4 : évolution du poids en fonction du périmètre thoracique chez les femelles $(n=215)$. 
d'équation a été proposé chez le zébu Gobra sénégalais (4) et le taurin Kapsiki du Cameroun (6). La mesure du périmètre thoracique peut être utilisée sans une deuxième mensuration telle que la hauteur au garrot qui n'améliore que très peu la précision des estimations (16). L'amélioration de la précision apportée par la hauteur au garrot ne devient sensible que chez des animaux adultes (10). L'équation basée uniquement sur le périmètre thoracique a l'avantage d'être simple, de demander moins de travail et de permettre l'établissement de tables de conversion du périmètre thoracique en poids directement applicables (15).

En Côte d'Ivoire, ces tables ont été regroupées dans un Atlas de barymétrie qui a été distribué aux agents sur le terrain. Sur les fiches de suivi de certains programmes de recherche, c'est le périmètre thoracique qui figure à défaut de la pesée et la conversion est réalisée à l'aide d'un ordinateur. La nature des données est mentionnée (mensurations ou pesées) pour permettre d'évaluer le degré de précision ou d'erreur (10).

Les différentes équations barymétriques envisagées chez le zébu Azawak ont concerné des animaux essentiellement jeunes. Chez les animaux adultes, la précision devient insuffisante du fait du niveau d'engraissement ou de l'état physiologique chez les femelles reproductrices (10). Les états musculaires et d'engraissement modifient de façon très sensible la nature des liaisons observées. En particulier les dépôts adipeux obéissent à des lois très différentes de celles selon lesquelles s'opère la croissance des autres tissus (16).

\section{CONCLUSION}

Les formules barymétriques proposées chez le zébu Azawak sont des régressions polynomiales établies sur la base du périmètre thoracique et de la longueur scapulo-ischiale. Elles peuvent être utilisées, avec une précision très satisfaisante, lors du suivi pondéral d'animaux en croissance. Dans le cadre de la diffusion et du suivi des performances pondérales du zébu Azawak, certaines mensurations (périmètre thoracique et longueur scapulo-ischiale), à l'avenir, devront être considérées.

\section{Remerciements}

Nous exprimons notre reconnaissance au Pr G. Torreele pour son aide dans le traitement statistique des données.

\section{BIBLIOGRAPHIE}

1. ACHARD F., CHANONO M., 1995. Un système d'élevage performant bien adapté à l'aridité à Toukounous, dans le Sahel nigérien. Sécheresse, $2: 215-222$.

2. ACHARD F., CHANONO M., 1997. Mortalité et performances de reproduction chez le zébu Azawak à la station de Toukounous, Niger (1986-1992). Revue. Elev. Méd. vét. Pays trop., 50 : 325-333.

3. BARTHA R., 1968. Elevage de zébu Azawak à la station de Toukounous (Rapport 1963-1967), République du Niger. Niamey, Niger, ministère de l'Agriculture, $55 \mathrm{p}$.

4. CHOLLOU M., DENIS J.P., GAUCHET D., 1978. Calcul d'une formule barymétrique adaptée au zébu Gobra. Revue Elev. Méd. vét. Pays trop., $31: 447-450$.

5. CIPEA, 1978. Evaluation des productivités des races bovines Maure et Peul à la station du Sahel. Niono, Mali, Cipea, p. 91-97. (Monographie $\left.n^{\circ} 1\right)$

6. DINEUR B., THYS E., 1986. Les Kapsiki : race taurine de l'extrêmeNord camerounais. I. Introduction et barymétrie. Revue Elev. Méd. vét. Pays trop., 39 : 435-442.

7. DOMINGO A.M., 1976. Contribution à l'étude de la population bovine des états du golfe du Bénin. Paris, France, Agence de coopération culturelle et technique, $143 \mathrm{p}$.

8. FALL A., DIOP M., SANDFORD J., WISSOCQ Y.J., DURKIN J., TRAIL J.C.M., 1982. Evaluation des productivités des ovins Djallonké et des taurins N'Dama au Centre de recherches zootechniques de Kolda, Sénégal. Addis Abeba, Ethiopie, Cipea, 74 p. (Rapport de recherche n 3)

9. IRAHIMA Z., 1986. L'élevage des bovins, ovins et caprins au Niger. Etude ethnologique. Thèse Doct. vét., EISMV, Dakar, Sénégal, 111 p.
10. LANDAIS E., 1983. Analyse des systèmes d'élevage bovin sédentaire du nord de la Côte d'Ivoire. Tome II. Données zootechniques et conclusions générales. Maisons-Alfort, France, Gerdat-lemvt, p. 411-431.

11. LARRAT R., PAGOT J., VAN DEN BUSSCHE J., 1985. Manuel des agents techniques de l'élevage tropical. Maisons-Alfort, France, lemvtCirad, p. 466-468.

12. MARMET R., 1983. La connaissance du bétail. Tome 1. Les bovins, $2^{\mathrm{e}}$ éd. Paris, France, Technique et documentation, p. 85-92.

13. PAGOT J., 1985. L'élevage en pays tropicaux. Paris, France, Maisonneuve et Larose/Agence de coopération culturelle et technique, p. 372-375. (Coll. Techniques agricoles et productions tropicales)

14. PAGOT J., DELAINE R., 1959. Etude biométrique de la croissance des taurins N'Dama. Revue Elev. Méd. vét. Pays trop., 12 : 405-416.

15. PLANCHENAULT D., 1987. Essai d'amélioration génétique des bovins en milieu défavorable. Exemple du ranch de Madina-Diassa au Mali. Thèse Doct., Université Pierre et Marie Curie, Paris/Maisons-Alfort, France, Cirad-lemvt, $307 \mathrm{p}$

16. POIVEY J.P., LANDAIS E., SEITZ J.L., 1980. Utilisation de la barymétrie chez les races taurines locales de Côte d'Ivoire. Revue Elev. Méd. vét. Pays trop., $33: 311-317$.

17. SOW R.S., DENIS J.P., TRAIL J.C.M., THIONGANE P.I., MBAYE M., 1991. Note sur la barymétrie comme moyen de sélection indirecte du poids vif chez le zébu Gobra au Sénégal. Revue Elev. Méd. vét. Pays trop., $4 \mathbf{4}:$ 97-100.

18. SYMOENS C., HOUNSOU VE G., 1991. Mesures barymétriques chez le bétail Borgou dans le Nord-Est Bénin. Revue Elev. Méd. vét. Pays trop., 44 : 487-490.

Reçu le 30.03.2001, accepté le 03.09.2001 


\section{Summary}

Dodo K., Pandey V.S., Illiassou M.S. Weight Estimation from Body Measurements of the Azawak Zebu in Niger

The objective of this study was to establish weight estimation equations from body measurements applicable to the Azawak zebu. The weight and 12 types of measurements were recorded in 343 Azawak zebus at the Sahelian experimental station of Toukounous in Niger. Most of the animals were young: $74 \%$ were at most two years old. Out of the 12 types of measurements, three were retained because of their correlation with the body weight (thoracic perimeter, height at the withers and scapulo-ischial length). The weight correlation was higher with the thoracic perimeter $(r=0.97)$ and the scapulo-ischial length $(r=0.96)$ than with the height at the withers $(r=0.92)$. Several types of equations between the weight and two types of measurements (thoracic perimeter and scapulo-ischial length) were compared. The selected equation for all the animals (males and females) was a polynomial regression of the predicted weight $(y)$ over the thoracic perimeter $(x)$ and the scapulo-ischial length $(z)$ : $y=$ $0.02697 x^{2}-4.25 x+1.535 z+96.145$ with a coefficient of determination $r^{2}=0.989$ and a residual standard deviation $\left(\mathrm{S}_{\mathrm{y}}\right)$ of $11.23 \mathrm{~kg}$. The error between the observed and predicted weights, expressed as percentages of the observed weight, was lower than $5 \%$ in $57.2 \%$ of the animals and lower than $10 \%$ in $90.1 \%$ of them. For the males, an equation also with two variables was selected: $y=0.0260 x^{2}-3.856 x+$ $1.211 z+96.114$ with $r^{2}=0.990$ and $S_{y}=10.33 \mathrm{~kg}$. For the females, a regression only using the thoracic perimeter $(y=$ $\left.0.0281 x^{2}-3.294 x+125.217\right)$ showed great accuracy $\left(r^{2}=\right.$ 0.981 and $S_{y}=15.28 \mathrm{~kg}$ ). This latter equation was used to establish a conversion table of thoracic perimeters into body weights.

Key words: Cattle - Azawak zebu - Body measurement - Body weight - Niger.

\section{Resumen}

Dodo K., Pandey V.S., Illiassou M.S. Uso de la barimetría para la estimación del peso en el cebú Azawak en Níger

El objetivo del presente estudio fue el de determinar las ecuaciones barimétricas aplicables al cebú Azawak. Para esto, se obtuvieron los pesos, así como 12 tipos de medidas en 343 cebúes Azawak durante tres meses, en la estación experimental de Toukounous, en Níger. Los animales eran esencialmente jóvenes (74\% tenían menos de dos años de edad). Entre los 12 tipos de medidas, tres (el perímetro toráxico, la altura al garrote y la distancia escápulo-isquiática) se retuvieron para establecer la barimetría, debido a su correlación con el peso. La correlación del peso con el perímetro toráxico $(r=$ $0,97)$ o con la distancia escápulo-isquiática $(r=0,96)$ fue más elevada que con la altura al garrote $(r=0,92)$. Se compararon varios tipos de relación entre el peso y los dos tipos de medidas (perímetro toráxico y distancia escápulo-isquiática). La ecuación barimétrica que se retuvo para el conjunto de los animales (machos y hembras) fue una regresión polinominal de tipo predicción (y) sobre el perímetro toráxico (x) y la distancia escápulo-isquiática (z): y $=0,02697 x^{2}-4,25 x+$ $1,535 z+96,145$, con un coeficiente de determinación $r^{2}=$ 0,989 y una desviación estándar residual $\left(\mathrm{S}_{\mathrm{y}}\right)$ de 11,23 kg. El error entre el peso observado y el peso esperado, expresado en porcentaje del peso observado, fue inferior a $5 \%$ en $57,2 \%$ de los animales e inferior a $10 \%$ en $90,1 \%$ de ellos. Se propone igualmente una ecuación, también de dos variables, para los machos: $y=0,0260 x^{2}-3,856 x+1,211 z+96,114$ con $r^{2}=0,990$ y $S_{y}=10,33 \mathrm{~kg}$. En las hembras, una regresión únicamente a partir del perímetro toráxico $\left(y=0,0281 x^{2}-\right.$ $3,294 x+125,217)$ permitió igualmente la obtención de una buena precisión $\left(\mathrm{r}^{2}=0,981\right.$ y $\left.\mathrm{S}_{\mathrm{y}}=15,28 \mathrm{~kg}\right)$. Esta última ecuación se utilizó para establecer una tabla de conversión del perímetro toráxico en peso.

Palabras clave: Ganado bovino - Cebú Azawak - Medición del cuerpo - Peso corporal - Níger. 